\title{
NAGOYA
}

\section{MATHEMATICAL JOURNAL}

Volume 231

September 2018

Published by

Cambridge University Press on behalf of

Foundation Nagoya Mathematical Journal 


\section{NAGOYA \\ MATHEMATICAL JOURNAL}

\section{Editors}

de Bouard, Anne

École Polytechnique, CMAP, Palaiseau

Gallagher, Isabelle

Université Paris-Diderot

Henniart, Guy

Université Paris-Sud

Hesselholt, Lars (Managing Editor)

Nagoya University

Hirachi, Kengo

University of Tokyo

Honda, Ko

University of California, Los Angeles

Iyama, Osamu

Nagoya University

Kedlaya, Kiran

University of California, San Diego

Kondo, Shigeyuki

Nagoya University

Takahashi, Ryo

Nagoya University

Yoshida, Ken-ichi

Nihon University, Tokyo

\section{Associate Editors}

Besson, Gerard

Institut Joseph Fourier, Grenoble

Bona, Jerry L.

University of Illinois at Chicago

Fukaya, Kenji

Simons Center for Geometry and Physics, Stony Brook

Mori, Shigefumi

RIMS, Kyoto University

Mukai, Shigeru

RIMS, Kyoto University

Noguchi, Junjiro

University of Tokyo

Shoji, Toshiaki

Tongji University, Shanghai

Tamagawa, Akio

RIMS, Kyoto University 


\title{
NAGOYA \\ MATHEMATICAL JOURNAL
}

\author{
Volume 231
}

September 2018

\section{CONTENTS}

Esterov, A. and Takeuchi, K.: On vanishing theorems for local systems associated to Laurent polynomials...................

Àlvarez Montaner, J. and Yanagawa, K.: Lyubeznik numbers of

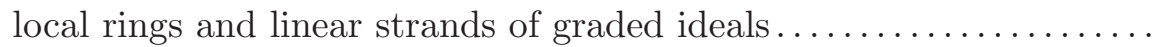

Burstall, F., Hertrich-Jeromin, U. and Rossman, W.: Discrete linear Weingarten surfaces

Ringel, C. M.: Lattice structure of torsion classes for hereditary artin algebras

Liu, H.: Singular integrals supported by subvarieties for

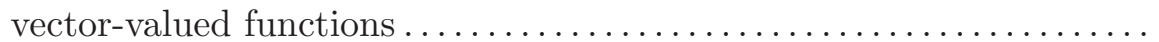

Martínez Torres, D., Del Pino, Á. and Presas, F.: The foliated Lefschetz hyperplane theorem $\ldots \ldots \ldots \ldots \ldots \ldots \ldots \ldots \ldots \ldots \ldots \ldots \ldots \ldots$

Oh, Y.-G. and Wang, R.: Analysis of contact Cauchy-Riemann maps II: canonical neighborhoods and exponential convergence for the Morse-Bott case 
